\title{
Performance et circuit de commercialisation des principaux produits agricoles des cuvettes oasiennes du département de Gouré (Niger)
}

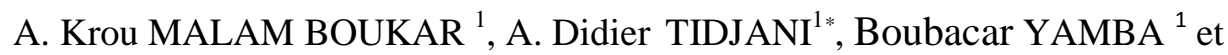 \\ Philippe LEBAILLY ${ }^{2}$ \\ ${ }^{1}$ Université Abdou Moumouni de Niamey. \\ ${ }^{2}$ Université de Liège (Belgique). \\ *Auteur correspondant ; E-mail : didierta@yahoo.fr ; Tel : (00227) 96997262.
}

\section{RESUME}

Les cuvettes oasiennes du département de Gouré sont sources de production alimentaire et de revenus pour les populations locales. L'objectif de cet article est de démontrer la performance économique des produits agricoles de cuvettes. Pour ce faire, trois démarches méthodologiques complémentaires ont été adoptées : La première permet d'identifier les produits agricoles les plus rentables selon l'avis des producteurs, la seconde permet de déterminer les principaux produits agricoles de cuvettes à travers le "test de Kendall " et la troisième permet de calculer la rentabilité économique des produits à travers des indicateurs de performance. Les enquêtes réalisées auprès de 105 producteurs dans neuf cuvettes, montrent que toutes les productions sont rentables. Les producteurs indiquent que le manioc est la spéculation la plus importante du fait de sa mise en culture, de sa consommation et de sa conservation facile. Les deux autres tests montrent les cultures à cycle court comme le chou (75\%) et l'oignon $(69 \%)$ sont plus attrayants. Les produits agricoles de cuvettes sont plus destinés à la vente $(73 \%)$ qu'à l'autoconsommation $(27 \%)$ et les marchés locaux représentent les principales plateformes commerciales. Ces constats montrent que malgré l'opportunité qu'offrent ces cuvettes oasiennes, des défis restent à relever.

(C) 2016 International Formulae Group. All rights reserved.

Mots clés: cuvette oasienne, performance, commercialisation, Gouré, Niger.

\section{Performance and marketing channel of the main agricultural products of the oasis basins in the department of Goure (Niger)}

\begin{abstract}
The oasis basins of Gouré stand for food production and income generating sources for the local populations. The objective of this study is to show the economic performance of the main agricultural basins products. Therefore, two important complementary methodological approaches have been considered. The first helps in identifying the main agricultural products from basins through "test of Kendal" and the second allows the calculation of the economic profitability of the products through some indicators for measuring their
\end{abstract}


performance. The investigations had realized in January 2015 among 105 producers. The analysis of results were recorded which revealed that all the agricultural products are generating some profit. However, the profit earning capacity is more important for short cycle products like cabbage (75\%) and onions (69\%). Basins products are more likely sold (73\%) than being used for self-consumption (27\%) and local markets remain the major business centers. These findings show that despite the opportunity offered by these oasis basins, challenges remain.

(c) 2016 International Formulae Group. All rights reserved.

Keywords: oasis basin, performance, marketing, Goure, Niger.

\section{INTRODUCTION}

Les cuvettes oasiennes du Sud-Est nigérien disposent d'énormes potentialités en matière de production agricole. Ce potentiel varie suivant la localisation de la cuvette qui impose le niveau de nappe phréatique (Ambouta et al., 2005). Ainsi, la mise en spécificité des cuvettes oasiennes est d'un intérêt croissant au regard de la place qu'occupe les productions agricoles dans la sécurisation des ménages agricoles (Awa Krou, 2016 in Jahiel, 1998). Du fait de la baisse généralisée de la production agricole suites aux effets combinés du changement climatique (Ozer et al., 2005) et de la croissance démographique (INS, 2012), les populations vivant à proximité des cuvettes déploient l'essentiel de leur effort dans l'exploitation des cuvettes pour pallier au déficit lié à la baisse de la production de la culture pluviale. Pour mieux appréhender cette valorisation qui constitue une bonne stratégie d'adaptation au changement climatique, il est important de comprendre l'importance de la production agricole dans les cuvettes et de mesurer l'efficacité économique des principales spéculations agricoles suivant les types de cuvettes et la proximité des centres commerciaux. L'objectif principal de cet article est de déterminer l'importance socioéconomique des productions agricoles issues des cuvettes oasiennes.

\section{MATÉRIEL ET MÉTHODES \\ Zone d'étude}

La zone d'étude correspond à la zone d'intervention du Projet de Lutte contre l'Ensablement des Cuvettes Oasiennes (PLECO) dans le département de Gouré. Neuf (9) cuvettes faisant office de centre d'intérêt du Programme Inter-universitaire CibléRecherche sur l'Ensablement des Cuvettes $2^{\text {ième }}$ phase (PIC/REC II) ont servi de support à cette étude. Parmi elle, trois sont à eau affleurante, trois à eau intermédiaire et trois à eau profonde. Les critères de classification des dites cuvettes sont le niveau de la nappe phréatique, la typologie et le type d'exploitation (Tableau 1). Selon le Recensement Général de l'Agriculture et du Cheptel (RGAC) de 2008, les 9 cuvettes sélectionnées totalisent 1056 ménages agricoles et les enquêtes de terrain menées en 2012 ont porté sur 105 exploitants soit 10\% de l'ensemble $(\mathrm{Ce}$ recensement définit «un ménage agricole comme un ménage où au moins un membre cultive ou pratique de l'élevage pour son propre compte ou le compte du ménage).

\section{Enquête socioéconomique}

L'enquête socio-économique réalisée auprès des 105 exploitants a porté essentiellement sur la perception des exploitants sur les spécificités des cuvettes oasiennes du département de Gouré, la perception des exploitants sur l'importance des produits agricoles, la hiérarchisation des 
produits agricoles, la structure des prix des produits agricoles de cuvette, l'analyse des indicateurs de rentabilité économique, ainsi que les acteurs de la production et de la commercialisation des produits agricoles de cuvettes.

\section{Traitement des données collectées}

Les données collectées sont traitées avec les logiciels Sphinx Plus ${ }^{2}$, Excel et Arc gis 10 .

\section{RESULTATS}

Perception des exploitants sur les spéculations agricoles les plus rentables dans les cuvettes oasiennes de Gouré

Respectivement 8, 10 et 10 groupes de spéculations sont faites dans les cuvettes à eau profonde, intermédiaire et affleurante. La Figure1 montre les spéculations agricoles les plus rentables dans les cuvettes de Gouré. Selon l'avis des producteurs, il ressort que dans les cuvettes à eau affleurante et intermédiaire, la canne à sucre et le manioc constituent les spéculations les plus rentables, tandis que dans les cuvettes à eau profonde, ce sont le manioc et le chou qui occupent ces places. La diversité des spéculations est fortement conditionnée par la qualité du sol et la disponibilité en eau. Ainsi, les cultures les plus exigeantes en eau comme la canne à sucre et la banane s'observent au niveau des cuvettes à eau affleurante et à eau intermédiaire et les moins exigeantes représentées par les autres spéculations de la Figure 1 au niveau des trois types de cuvettes. Au niveau de la mise en œuvre de ces cultures, il faudrait noter que celle du manioc et de la canne à sucre n'exigent pas beaucoup de travaux supplémentaires après la plantation. Cependant, bien qu'elles soient à cycle long ces spéculations génèrent des revenus socioéconomiques d'où la perception qu'ont les paysans de leur bonne rentabilité. Les autres spéculations, bien qu'elles soient de cycle court sont exigeantes autant en temps de travail qu'en exigence en intrant agricole, d'où la faible perception des revenus qu'elles génèrent. L'arboriculture fruitière, bien facile à mettre en œuvre n'est pas bien développée dans les cuvettes, malgré le potentiel qu'ils regorgent, d'où la faible perception des revenus qu'il génère. Les autres spéculations représentent les spéculations exotiques dont l'exploitation n'est pas généralisée.

La Figure 2 montre une compilation des spéculations les plus rentables faites en combinant les résultats obtenus au niveau de toutes les cuvettes étudiées selon les exploitants. Le manioc occupe la première place, la seconde place est occupée par le groupe canne à sucre et chou, le troisième groupe est représenté par l'oignon, la patate douce, la banane et le maïs. La pomme de terre, l'arboriculture et autres cultures de moindre importance ferme la marche.

Pour la suite de ce travail, les 5 spéculations les plus rentables à savoir (1) le manioc (Manihot esculenta); (2) la canne à sucre (Saccharum officinarum) ; (3) le chou (Brassica oleracea); (4) l'oignon (Allium сера) et (5) la patate douce (Ipomoea batatas), ont été retenues (Figure 2) pour l'analyse de la performance économique.

\section{Hiérarchisation des produits agricoles de cuvette selon le "Test de Kendall"}

Le classement fourni par les exploitants répond à la logique paysanne qui consiste à considérer comme rentabilité le revenu brut de l'exploitation (RBE). Or, ce dernier ne prend pas en compte tous les autres aspects des charges intermédiaires en particulier la main d'œuvre familiale, les amortissements, etc. C'est pourquoi, il est nécessaire de soumettre ces résultats au "test de kendall". Dans toutes les 9 cuvettes étudiées, les principales spéculations ont été soumises au test de Kendall, afin de tester la concordance des classements effectués par les différents groupes d'entretien. A la lumière des résultats de ce test (Tableau 3), les classements 
effectués par les exploitants sont loin d'être cohérents, car certains aspects du processus d'exploitation/production, ne sont pas pris en compte, d'où la nécessité de leur vérification à travers le "test de kendall".

Le "test de kendall " classe l'oignon comme la culture la plus rentable sur les 5 principales spéculations (N). Il est suivi du chou et de la patate douce. On constate selon ce test, que les cultures à cycle court se révèlent plus rentables par rapport aux cultures à cycle long notamment le manioc et la canne à sucre.

\section{Structure des prix des principaux produits} agricoles de cuvette ( $\mathrm{Fcfa} / \mathrm{kg}$ )

L'analyse des effets au prix du producteur et au prix du marché complète l'approche socio-économique en mesurant les effets indirects de l'exploitation des cuvettes oasiennes. L'efficacité économique du système d'exploitation est ici fondée sur la comparaison des coûts nécessités pour une unité de produit. Le Tableau 4 montre les détails sur le coût total de production d'un kilogramme (y compris le coût de la main d'œuvre familiale) de chaque principale spéculation.

Les données du Tableau 4 décrivent la structure fonctionnelle des prix des principales spéculations au cours de la campagne 2013. Les prix observés sur le marché de Soubdou varient dans le temps et est régi par la loi de l'offre et de la demande (Soubdou est le principal marché d'écoulement des produits agricoles de cuvette. Il est situé sur la RN1, à $38 \mathrm{~km}$ à l'est de la ville de Gouré). La Figure 3 montre deux grandes phases dans la variation des prix des produits maraîchers de deux périodes : Une première période allant du mois d'octobre à juin correspondant à la saison sèche et où les prix des produits sont moins élevés à cause de l'intensification des activités dans les cuvettes et une deuxième période (juillet-août et septembre) correspondant à la saison des pluies et où les prix connaissent une hausse vertigineuse. Cette hausse s'explique par la diminution des activités maraîchères, car les exploitants sont en ce moment occupés par les travaux champêtres. Cette remontée sur le front dunaire réduit considérablement la présence des exploitants dans les cuvettes. Par voie de conséquence, la production diminue dans les cuvettes et les coûts se renchérissent sur les marchés.

\section{Analyse des indicateurs de rentabilité économique}

L'établissement des comptes consolidés par sous-filière met en évidence, d'une part la distribution de la valeur ajoutée générée par chaque spéculation et d'autre part la rentabilité privée nette (RPN) des exploitations (Tableau 5).

L'analyse économique des principales spéculations permet de tirer les conclusions suivantes :

- Pour toutes les 5 principales spéculations, la RPN est positive $(+50 \%)$. Autrement dit, le bénéfice obtenu de chaque spéculation dépasse de moitié le capital investi.

- La rentabilité est plus attrayante pour l'oignon $(69 \%)$ et pour le chou $(65,47 \%)$, car les charges intermédiaires sont moins élevées. Le fait qu'elles soient des cultures à cycle court, offre la possibilité de les pratiquer 2 à 3 fois par an, alors que le manioc et la canne à sucre dont les cycles de production nécessitent des charges plus importantes au moment de la plantation, les RPN sont par conséquent moins élevées.

\section{Analyse spatiale du circuit de commercialisation des principaux produits agricoles de cuvette}

La commercialisation des produits agricoles peut se définir comme l'accomplissement de toutes les activités commerciales s'exerçant dans le mouvement des biens et la prestation des services depuis 
le point initial de la production agricole jusqu'au stade ultime où les produits arrivent entre les mains du consommateur (Kohls, 1961, in FAO. 2007). De ce fait, le passage de la production à la commercialisation est un acte important en matière agricole (Diemer, 2015).

Les produits des cuvettes oasiennes du département de Gouré sont essentiellement écoulés sur les marchés locaux dont les plus importants sont les marchés hebdomadaires de Soubdou et de Guidiguir. Ce sont des véritables carrefours d'échanges pour les oasiens. Leur position géographique qui les place au centre du département et sur la RN1 facilitent toutes trasactions. En effet, plus de $57 \%$ des quantités physiques produites dans les cuvettes oasiennes sont écoulées dans ces deux marchés. Il s'agit essentiellement des fruits et légumes $(47,5 \%)$, les tubercules $(37,8 \%)$ et les autres produits comme la laitue, la tomate, le gombo représentent 14,6\%. En outre, presque $1 / 3$ de la production est acheminée en direction des marchés intérieurs $\mathrm{du}$ pays notamment Zinder, Madaoua et Agadez. Ce circuit concerne le manioc, la canne à sucre et les palmiers doum. Enfin, la commercialisation du natron est assurée par le circuit sous-régional en particulier les plateformes commerciales du Nigeria notamment Machina et Maigatari. La Figure 4 présente la dimension spatiale de la plateforme commerciale des produits des cuvettes oasiennes du département de Gouré.

\section{Analyse des relations entre les acteurs de la commercialisation}

Plusieurs acteurs interviennent dans la chaîne la commercialisation des produits de cuvette (Figure 5) et cela à différents degrés d'intermédiation. Deux grandes chaînes sont ainsi distinguées : la chaîne de production et la chaîne de distribution. Les agents économiques clés sont repartis entre ces grandes chaînes. Pour la première, il s'agit essentiellement des producteurs ou exploitants et pour la deuxième chaîne, les intermédiaires, les grossistes, les transporteurs et les détaillants. En effet, l'exploitant est à l'origine de la première transaction commerciale des produits de cuvette. Son rôle est de produire et de disposer d'une offre en produits nécessaire et aux besoins de la demande des consommateurs. Toutefois, une partie de la production est autoconsommée par les producteurs eux-mêmes.

Pour la chaîne de distribution, le grossiste se place en tête de la chaîne. Il engage les moyens financiers et prend ainsi le risque de réaliser une marge bénéficiaire, étant donné la nature périssable de certains produits de cuvettes et l'incertitude liée au transport routier sur longue distance. Dans certains cas, le grossiste emploie des collaborateurs localement appelé «dilali » qui s'occupent de la location du camion, du paiement des frais opérationnels et qui sont responsables du chargement et de l'acheminement depuis les pôles productifs jusqu'aux marchés d'écoulement. $\mathrm{Au}$ regard du caractère sablonneux et l'isolement des zones de production, le grossiste emploie un guide local (imbiko) qui l'accompagne lors du voyage pour faciliter les transactions. Les intermédiaires appelés aussi «dilali» rémunérés par le grossiste, suivent la transaction entre la zone de production et le marché de destination finale du produit. Cette filière concerne essentiellement le natron écoulé au Nigeria. Les opérations de chargement des camions sont suivies par le «Serkin Tacha», le chef de ligne, responsable de la gestion des arrivées et départs depuis les autogares, qui reçoit un forfait pour son service. 
Tableau 1: Critères de sélection des cuvettes d'étude.

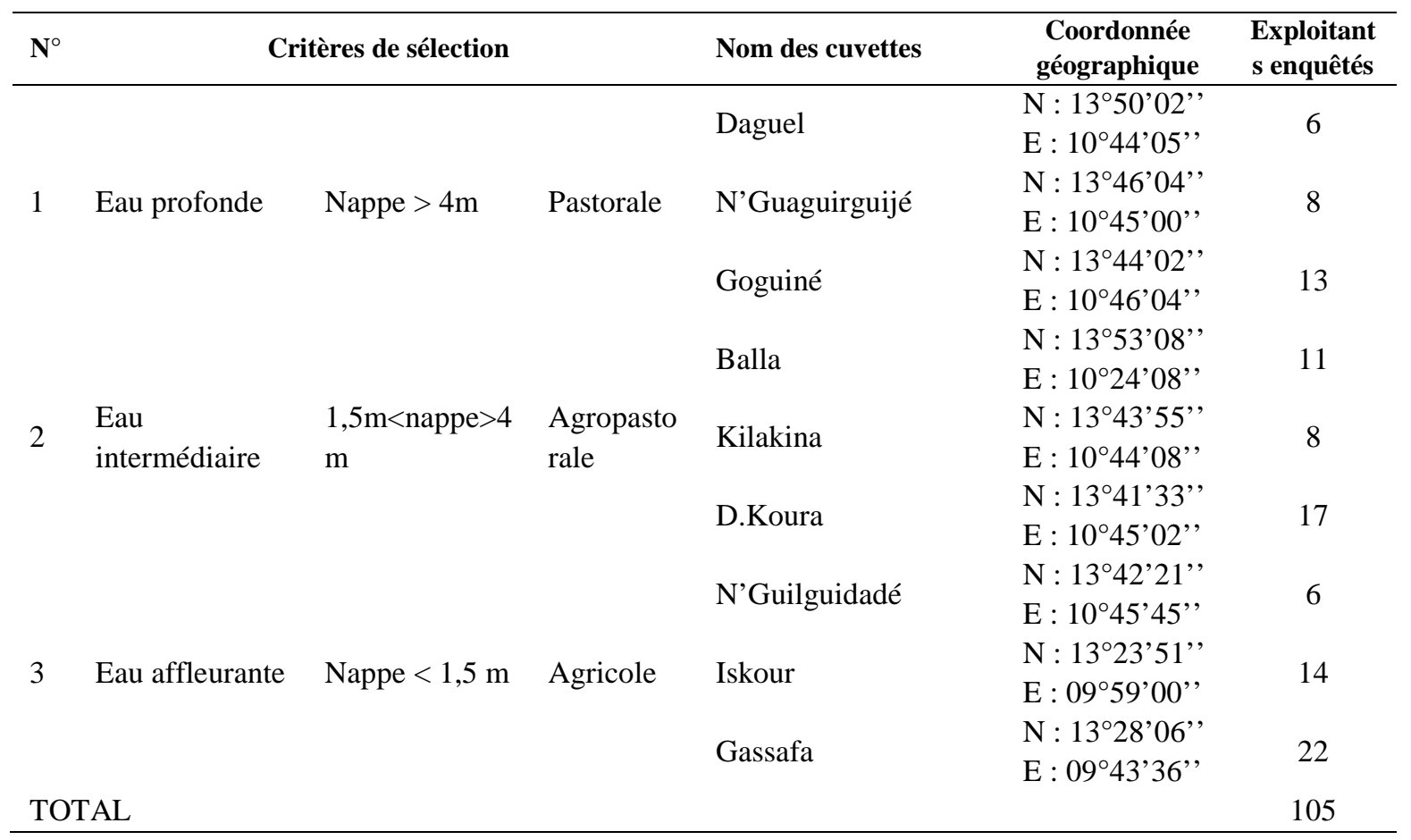

Tableau 2: Calcul des indicateurs de rentabilité (Fabré P. 1994).

\begin{tabular}{ll}
\hline Agrégats & Formules \\
\hline Produit Brut $(\mathrm{PB})$ & $P B=R d t^{*} P U$ \\
Consommations intermédiaires $(\mathrm{CI})$ & $C I=($ Rémunération du travail + Frais \\
& financiers + Taxes $)$ \\
Valeur ajoutée $(\mathrm{VA})$ & $V A=P B-C I$ \\
Amortissement $\left(\mathrm{Am} \mathrm{m}_{\mathrm{i}}\right)$ & $\left.\mathrm{Am} \mathrm{m}_{\mathrm{i}}=\left(\mathrm{n}_{\mathrm{i}}^{*} \mathrm{PU} \mathrm{PU}_{\mathrm{i}}\right) / \mathrm{D}_{\mathrm{i}}\right) *(\mathrm{p} / 100)$ \\
Revenu Brut de l'Exploitation $(\mathrm{RBE})$ & $R B E=V A-($ Travail + Frais financiers + Taxes $)$ \\
Rentabilité Privée Nette $(\mathrm{RPN})$ & $R P N=V A / P B$ \\
\hline
\end{tabular}

Tableau 3: Hiérarchisation des principales spéculations (Test de Kendall).

\begin{tabular}{|c|c|c|c|c|}
\hline Spéculations & Rang moyen & Ordre & \multicolumn{2}{|c|}{ Test de Kendall } \\
\hline Oignon & 1,38 & 1 & $\begin{array}{r}\mathrm{N} \\
\mathrm{W} d \mathrm{e}\end{array}$ & 5 \\
\hline Chou & 2,82 & 2 & Kendall & 0,39 \\
\hline Patate douce & 4,49 & 3 & Khi-deux & 16,83 \\
\hline Manioc & 4,50 & 4 & $\mathrm{dl}$ & 4 \\
\hline Canne à sucre & 7,01 & 5 & Probabilité & 0,00 \\
\hline
\end{tabular}


Tableau 4: Structure de prix des principaux produits agricoles (année 2014).

\begin{tabular}{|c|c|c|c|c|c|c|}
\hline \multirow[b]{2}{*}{ Spéculations } & \multicolumn{2}{|c|}{ Production } & \multicolumn{2}{|c|}{ Prix/unité (Fcfa/kg) } & \multirow[b]{2}{*}{ Prix moyen } & \multirow{2}{*}{$\begin{array}{c}\text { Produits } \\
\text { (en Fcfa/kg) }\end{array}$} \\
\hline & (en unité locale) & (en Kg) & $\begin{array}{l}\text { Prix au } \\
\text { producteur }\end{array}$ & $\begin{array}{l}\text { Prix au } \\
\text { marché }\end{array}$ & & \\
\hline Oignon & sac de $100 \mathrm{~kg}$ (292) & 29200 & 300 & 325 & 313 & 9125000 \\
\hline Chou & panier (1824) & 45600 & 60 & 85 & 73 & 3306000 \\
\hline Patate douce & sac de $100 \mathrm{~kg}(232)$ & 23308 & 130 & 150 & 140 & 3263120 \\
\hline Manioc & sac de $100 \mathrm{~kg}(955)$ & 50541 & 185 & 215 & 200 & 10108200 \\
\hline Canne à sucre & stère (604) & 30240 & 150 & 175 & 163 & 4914000 \\
\hline
\end{tabular}

Tableau 5: Calcul de la rentabilité économique d'1 ha par spéculation.

\begin{tabular}{lrrrrr}
\hline Indicateurs & \multicolumn{1}{l}{ Chou } & \multicolumn{1}{c}{ Manioc } & \multicolumn{1}{c}{$\begin{array}{c}\text { Canne à } \\
\text { sucre }\end{array}$} & Patate douce & \multicolumn{1}{c}{ Oignon } \\
\hline Produits & 1368000 & 3050000 & 2268000 & 1515000 & 1380000 \\
Consommation & & & & & \\
intermédiaire* (CI) & 335461 & 854011 & 982958 & 552622 & 457728 \\
Valeur ajoutée (VA) & 1032539 & 1195989 & 1285042 & 962378 & 1022272 \\
RBE & 697079 & 341978 & 1043953 & 373416 & 1664545 \\
Amortissement & 56791 & 145100 & 88524 & 37811 & 85526 \\
RNE & 640288 & 1196878 & 955430 & 335605 & 679019 \\
\hline Rentabilité privée net & & & & & \\
(RPN) & $\mathbf{6 5 , 4 7}$ & $\mathbf{6 2 , 8 0}$ & $\mathbf{5 6 , 6 5}$ & $\mathbf{6 3 , 5 2}$ & $\mathbf{6 9 , 0 0}$ \\
\hline
\end{tabular}

RBE : Revenu Brut de l'Exploitation ; RNE : Revenu Net de l'Exploitation

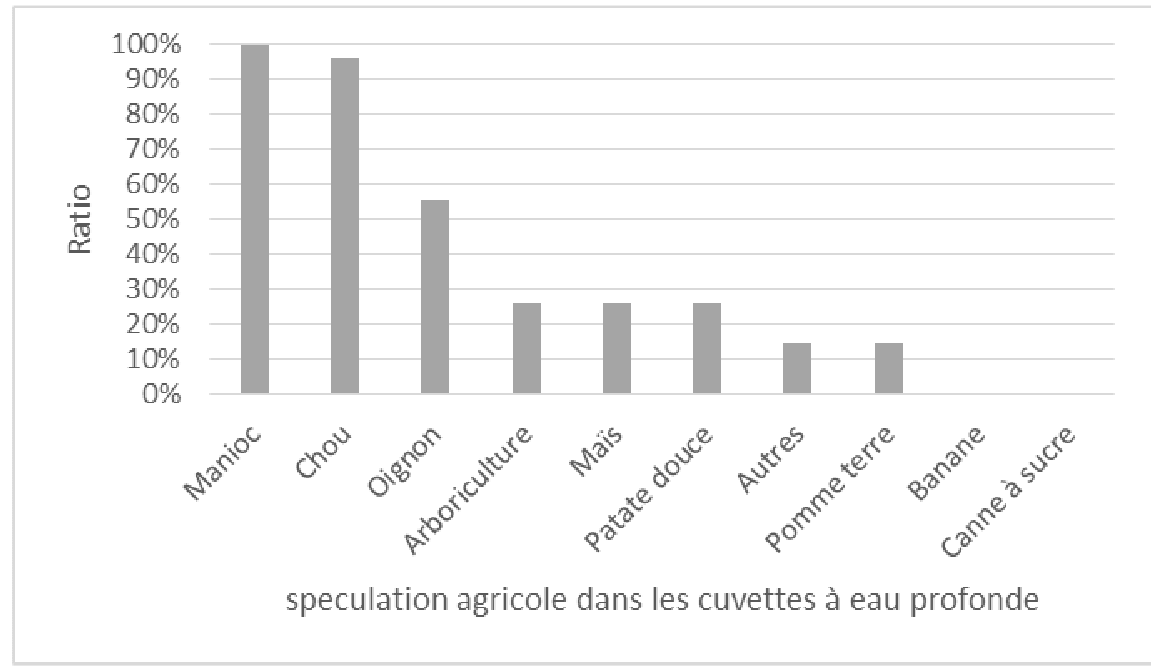


A. K. MALAM BOUKAR et al. / Int. J. Biol. Chem. Sci. 10(5): 2202-2214, 2016
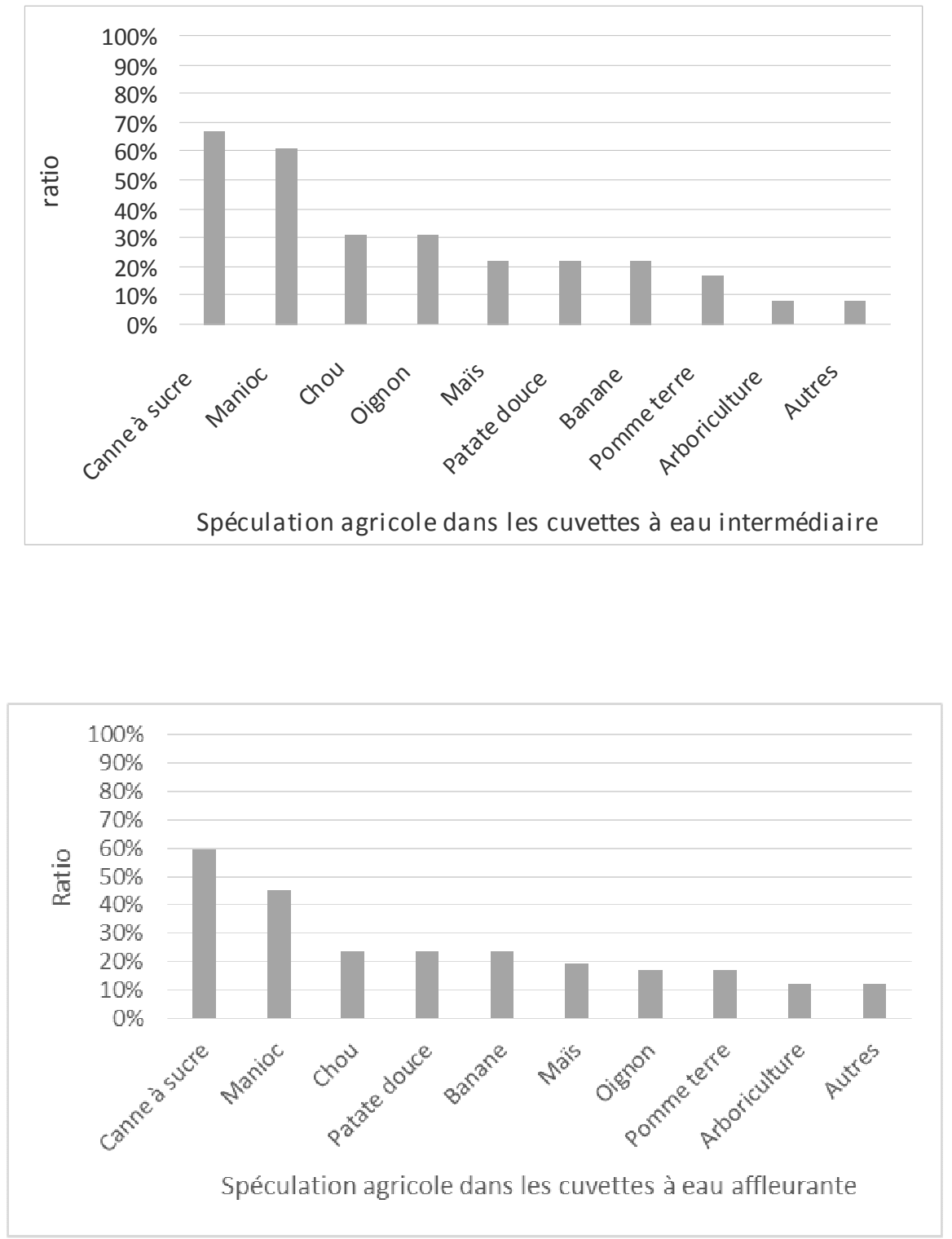

Figure 1: Spéculations agricoles les plus rentables selon les trois types de cuvette de Gouré. 
A. K. MALAM BOUKAR et al. / Int. J. Biol. Chem. Sci. 10(5): 2202-2214, 2016

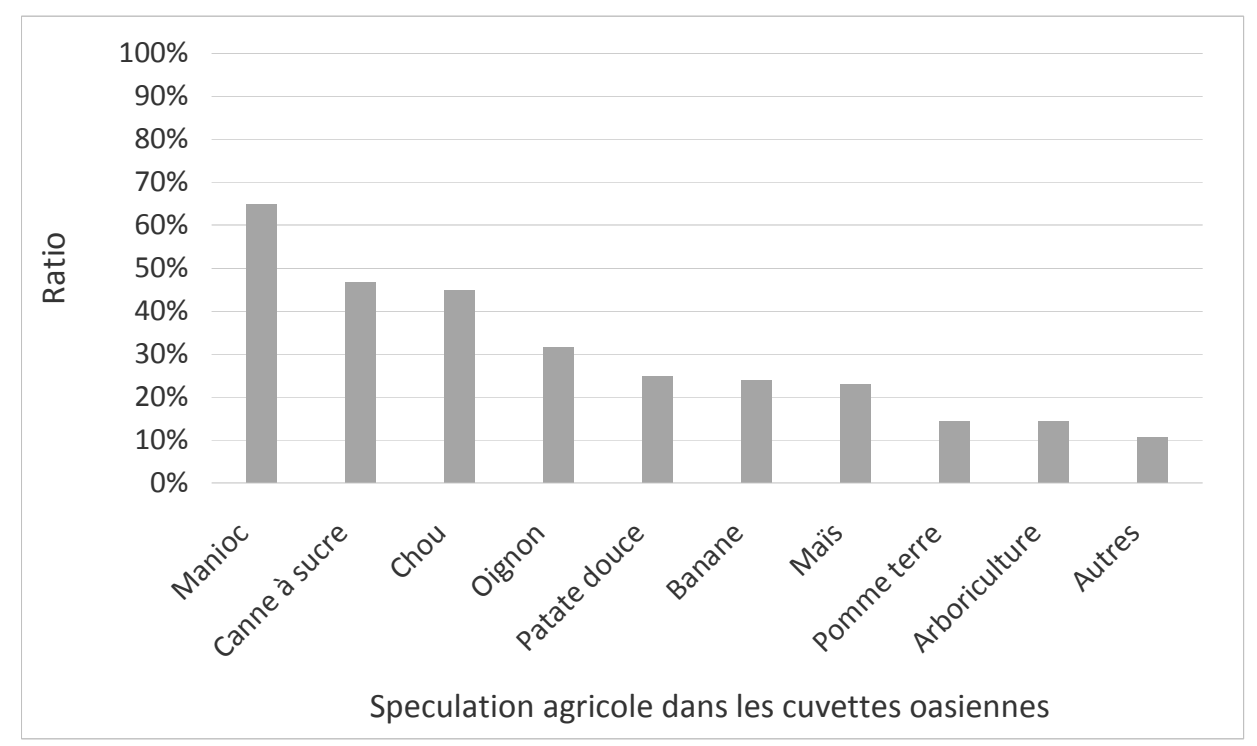

Figure 2: Classement des spéculations les plus rentables selon les exploitants.

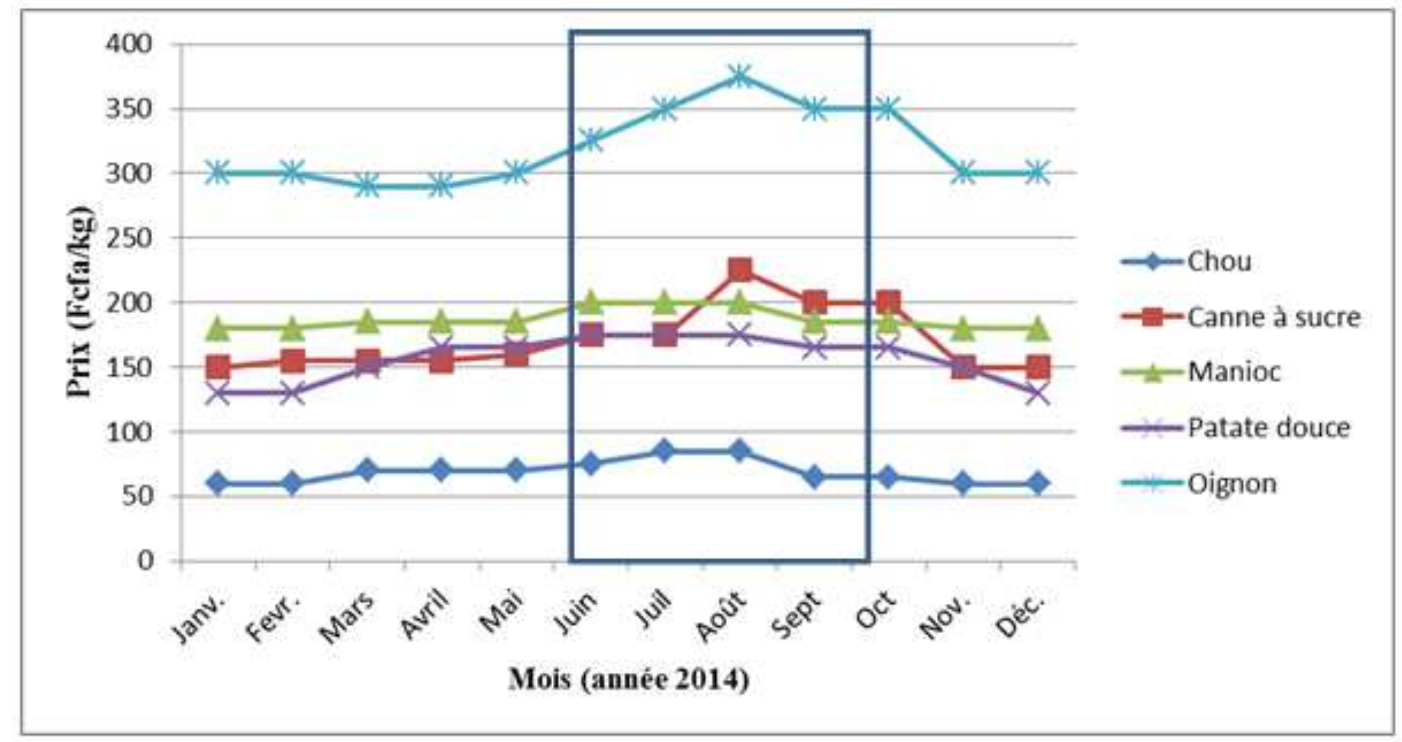

Figure 3: Variation des prix des produits de cuvette au marché de Soubdou en 2014. 


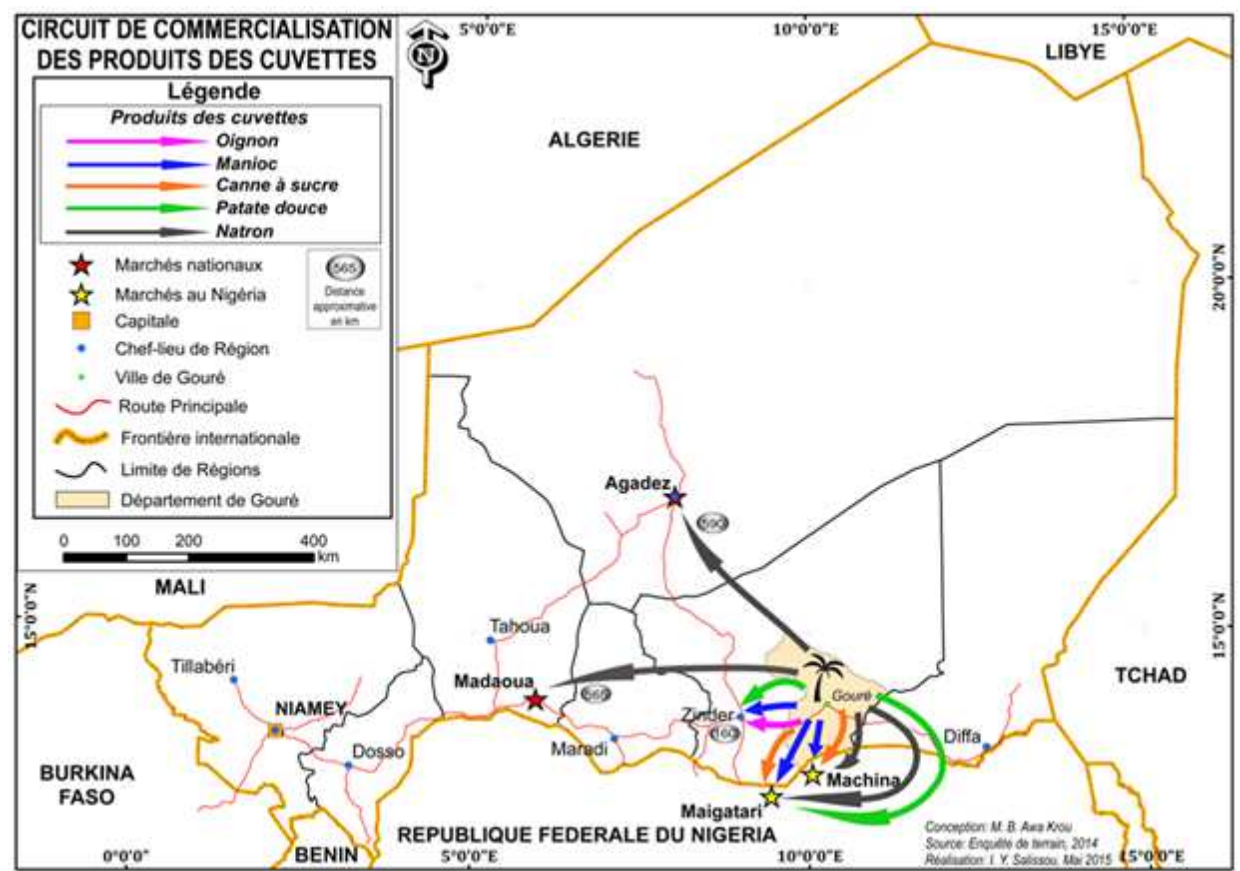

Figure 4: Zones de production et d'écoulement des principaux produits des cuvettes oasiennes de Gouré.

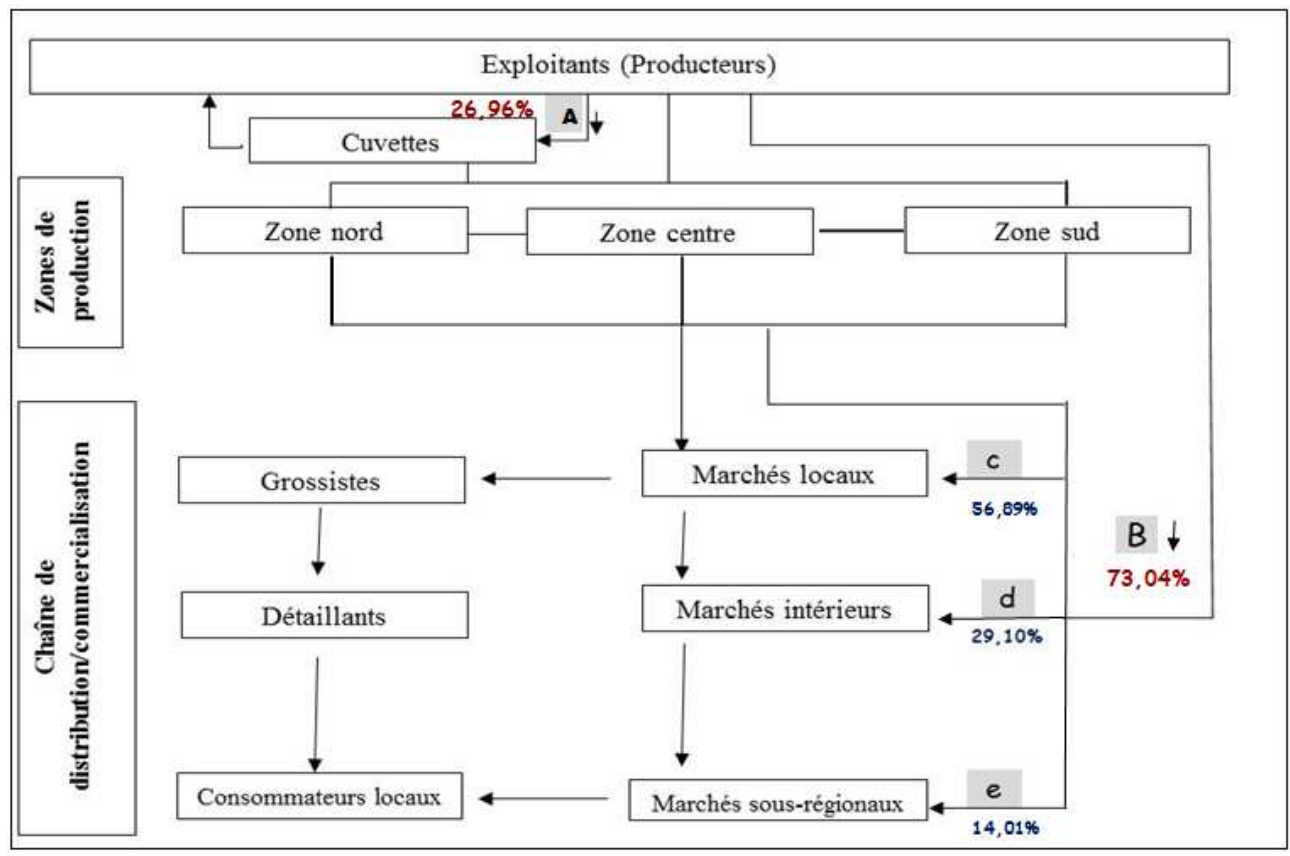

Figure 5: Diagramme de relation entre les acteurs de la commercialisation des produits de cuvettes.

A : Autoconsommation

B : Vente $(\mathrm{c}+\mathrm{d}+\mathrm{e})$

$\rightarrow$ Flux c: Distribution sur les marchés locaux [fruits et légumes $(47,50 \%)+$ Tubercules $(37,89 \%)$ et autres $(14,61 \%)$ ]

$\mathrm{d}$ : Distribution sur les marchés intérieurs [Tubercules et canne à sucre $(57,50 \%)+$ Natron $(23,00 \%)+$ palmes $(19,50 \%)$ ]

e : Distribution vers les marchés sous-régionaux [Natron $(59,10 \%)+$ Manioc et Canne à sucre $(40,90 \%)$ ] 


\section{DISCUSSION}

Les constats démographiques et socioéconomiques amènent à préciser que malgré l'opportunité économique qu'offrent les cuvettes oasiennes en matière de développement rural, les multiples contraintes à relever restent un défi majeur pour les années à venir (Tychon et al., 2009). Bien que les cuvettes constituent des sites de production agricole par excellence, la perception sur la rentabilité selon l'avis producteurs varie avec la rentabilité réelle calculée (Fabré, 1994), d'où la nécessité d'étudier toutes les dimensions de la rentabilité. En effet, la rentabilité selon les producteurs ne prend pas en compte les aspects liés aux consommations intermédiaires, d'où le classement du Manioc comme la spéculation la plus rentable. Cependant, le "test de kendall" classe les spéculations à cycle court comme le chou et l'oignon en tête, car les charges liées à leur mise en culture sont moindres par rapport au Manioc et la canne à sucre qui sont des cultures à cylce long. L'analyse de la filière de commercialisation révèle que les intermédiaires entre le producteur et le consommateur sont nombreux et contribuent à rendre plus cher le produit sur le marché. Cependant, dans la majorité des cas, surtout pour les cuvettes se trouvant à longue distance des marchés, les grands bénéficiaires ne sont que les transporteurs, les commerçants grossistes et ce, au détriment des producteurs. Parce qu'il s'agit des produits saisonniers, facilement périssables et que les producteurs sont obligés de vendre au prix imposé par les grossistes. Le transport des produits agricoles $\mathrm{du}$ lieu de production au lieu de vente constitue un maillon qui contribue à la cherté du produit. En effet, les transporteurs fixent les frais de transport selon la destination (Arnould, 2001, in Tartiani et al., 2013).
Dans le département de Gouré, les prix sont fixés en fonction de la difficulté d'accès aux zones de production généralement difficile d'accès à cause de l'ensablement. Dans le commerce des produits agricoles, le transport constitue un maillon qui contribue à la cherté du produit agricole sans pour autant que le producteur en profite du prix de vente élevé.

La marge bénéficiaire des producteurs est dictée par la loi de l'offre et de la demande des produits agricoles (Robbiati et al., 2012 in Tartiani et al., 2013). Si l'offre en produit est supérieure à la demande, la marge bénéficiaire du producteur diminue et inversement, si la demande est supérieure à l'offre, le producteur en tire plus bénéfice. De ce fait, les marges bénéficiaires des producteurs sont fortement conditionnées par la période de production qui suppose une période de disponibilité ou de manque du produit sur le marché. Ainsi, si la production est faite au moment des périodes de faible disponibilité du produit, la marge bénéficiaire sera importante et vice versa. C'est pourquoi, la mise en spécificité des cuvettes oasiennes du Sud-Est nigérien est d'un intérêt croissant au regard de la place qu'occupent ces produits agricoles de cuvettes dans la sécurisation des ménages.

\section{Conclusion}

Le développement des cuvettes oasiennes passe par une connaissance de l'efficacité économique de chaque produit agricole. L'étude a permis d'identifier les principaux produits agricoles selon le type de cuvette et a démontré l'efficacité économique de chaque produit agricole. Les différents maillons du circuit de commercialisation des dits produits sont également identifiés. La rentabilité économique des principales spéculations est vérifiée, car leur RPN est 
positive et dépasse $50 \%$. Toutefois, la rentabilité est plus attrayante pour les cultures à cycle court comme le chou (75\%) et l'oignon (69\%), en raison des charges intermédiaires moins importantes. Il faut également noter que les produits agricoles de cuvettes sont destinés plus à la vente $(73 \%)$ qu'à l'autoconsommation (27\%) et les marchés locaux représentent les principales plateformes commerciales. Il est d'ores et déjà recommander de soutenir la sensibilisation des populations locales sur les avantages comparatifs des principaux produits agricoles et surtout ceux à cycle court. De réfléchir sur comment optimiser les productions, réduire les charges liées au transport et à la conservation de façon à ce que le véritable gagnant soit le producteur et non l'intermédiaire.

\section{CONFLIT D'INTERETS}

Les auteurs de cet article déclarent avoir aucun conflit d'intérêts.

\section{CONTRIBUTIONS DES AUTEURS}

Dans cet article, notre principale contribution est de montrer d'une part, les méthodes de classification des produits agricoles de cuvettes et d'autre part, l'avantage comparatif des cultures à cycle court comme le chou et l'oignon sur les cultures à cycle long comme le manioc et la canne à sucre.

\section{REMERCIEMENTS}

Aux termes de ce travail, nous tenons à remercier l'Académie de Recherche et d'Enseignement Supérieur (ARES) Coopération Universitaire au Développement (CUD) de la Belgique qui a financé le Programme Inter-universitaire Ciblé / Recherche sur l'Ensablement des Cuvettes
(REC/PIC) phase II (2012 - 2016), dans lequel cette étude a été menée.

\section{RÉFÉRENCES}

Ambouta J-M, Guéro S. 2005. Etude sur l'inventaire et la caractérisation pédologique et hydraulique des cuvettes oasiennes dans le département de Gouré. Karthala et AFVP, Projet d'Appui à la Gestion des Ressources Naturelles PAGRN. Niger.

Awa Krou M. 2016. Les cuvettes oasiennes du Niger Oriental : entre pratiques foncières et cadre législatif. RISD, 4(6): 13p.

Boukari M. 2011. Impact de l'exploitation du palmier doum (Hyphaene thebaica) sur la vie socio-économique des populations de la zone oasienne du Sud-est du Niger : cas du village d'Adobour dans le département de Maini Soroa. Mémoire de Maitrise, Département de géographie, Université de Niamey, 78p.

Diemer A. 2015. Production et commercialisation des produits agricoles. Gestion économique du produit, dossier 2, Arnaud Diemer, IHEDREA, MCF Clermont-Ferrand, 24p.

Fabre P. 1994. Note de méthodologie générale sur l'analyse de filière. Document de formation pour la planification agricole $n^{\circ} 35$, Service de Soutien aux Politiques Agricoles, Division de l'Assistance aux Politiques, FAO, Rome, Italie.

Jahiel M, Morou A. 1990. Evolution géographique de la phoeniciculture en relation avec la désertification. In Les Systèmes Agricoles Oasiens, Dollé V, TOUTAIN G (eds). CIHEAM: Monpellier.

Pini G, Tarchiani V. 2007. Les systèmes de production agro-sylvo-pastoraux du 
Niger : Description et analyse. Working paper 22, Italie, Turin.

Pleco. 2006. Répertoire des cuvettes et basfonds de goure. Analyse Diagnostique Détaillée de la zone d'intervention du PLECO, Division de la Statistique et de la Cartographie Forestière (DSCF), 46p.

République du Niger. 2008. Recensement Général de l'Agriculture et du Cheptel. Productivité des Exploitations Agricoles, 8.
République du Niger. 2012. Recensement Général de la Population et de l'habitat (RGP/H)Tychon B. 2011. Présentation des cuvettes oasiennes et de leur rôle socio-économique dans le Sud-Est du Niger, Université de Liège/ Université de Niamey, Article, 2p.

Tychon B, Ambouta K, Ozer A. 1999. Quel avenir pour les cuvettes oasiennes dans le Niger oriental ? Geo. Eco. Trop., 33(III-VI): 12p. 